\title{
Verbal reaction times to hues vs hue names and forms vs form names'
}

BARRY GHOLSON AND RAYMOND H. HOHLE

INSTITUTE OF CHILD BEHAVIOR AND DEVELOPMENT

Two choice reaction time (RT) studies were carried out in which different classes of stimuli--hues us hue names in one study and forms $v$ s form names in the other--occurred in independent lists of 2, 4, and 6 equally probable alternatives. Mean choice RTs were faster for hue names and form names than for hues and forms with 4 and 6 alternatives, but no significant differences were found in the 2-stimulus lists. In both studies, significant list length by stimulus type (e.g., hues vs hue names) interactions indicated greater increase in mean RT with increases in list length for forms and hues than for form names and hue names. It was concluded that differential amounts of generalization of interference accounted for the interactions. Effects of experimental variables on inferred components of RT were also examined.

A series of studies reported over the past 50 years (e.g., Brown, 1915; Comalli, Wapner, \& Werner, 1962; Ligon, 1932; Lund, 1927; Schiller, 1966; Stroop, 1935, 1938) have shown that most $S$ can read through a list of hue names faster than they can identify a corresponding list of hues.

Brown (1915), whose methodology and stimulus materials were similar to the experiments that followed, used four different hues and the corresponding hue names in lists of 100 . The 100 hue chips were pasted on a large sheet of cardboard, and the corresponding hue names were printed in black ink on a sheet of the same size and color. College Ss responded verbally by reading the words or naming the colors as fast as possible, with total time recorded on a stop watch. After 12 days of practice the average time to read through the list of hues was $41.4 \mathrm{sec}$ while the corresponding time required for hue names was $29.3 \mathrm{sec}$. Since average color naming time did not decrease over the last few practice sessions, Brown concluded the difference in speed between the functions did not depend upon differential amounts of practice. He suggested further that distinct physiological processes were involved.

Lund (1927) replicated Brown's results and also investigated discriminability of his stimuli. In the discriminability study the Ss ran a pencil down lists of hues or hue names and picked out (but did not name) all of one specified stimulus, then all of another, etc. He suggested that hues were more discriminable than hue names because name finding took about twice as long as hue finding. This result led Lund to conclude that differences in naming time therefore could not be due to discriminability of the stimuli and he, in contrast to Brown, attributed differences in naming speed to differential amounts of pre-experimental practice. As evidence for his hypothesis he tested Ss between five and 19 years of age and found: (a) prior to age seven, hue naming was faster than hue name reading; and (b) a five-year-old $S$ showed consistent differences in favor of naming hues across 13 practice sessions. These results may have been due, however, simply to lack of reading skills in very young children.

Subsequent studies (Ligon, 1932; Stroop, 1935; Comalli et al, 1962; Schiller, 1966) have confirmed faster response rates to hue names than to hues, and several (Ligon, 1932; Comalli et al, 1962; Schiller, 1966) have shown that differential amounts of pre-experimental practice with the two types of stimuli probably does not account for the difference. In each of the latter studies age was an independent variable and, although response rate increased with age, the difference between rate of hue name reading and color naming remained constant (provided $S$ had learned to read). If differential learning or practice were responsible for different response rates to the two types of stimuli, stimulus type by age interactions would be expected, since these school-age Ss would be expected to have practiced reading much more than color naming.

Alternative interpretations of differences in response rates have been proposed by several investigators. Schiller (1966) suggested that hue names provide a more efficient medium for coding than hues: printed hue names are more redundant than hue chips; hence time required for perceptual processing is greater for hue chips. Another hypothesis is that nonverbal competing responses interfere with naming responses to hues (Stroop, 1935, 1938). The suggestion is that Ss have responded only verbally to words such as "red" or "'green," while many nonverbal conflicting responses have become associated with the corresponding hues.

In the first study reported here, differential response latencies to hues vs hue names-with stimulus uncertainty as a second independent variable-were obtained using conventional reaction time (RT) procedures; i.e., a stimulus (hue or hue name) was presented singly, and latency from stimulus onset to onset of the (verbal) response was recorded.

Also, since Kaswan and Young (1965) have provided evidence that differences in recognition threshold tend, at least under some conditions, to parallel choice RT differences, tachistoscopic recognition threshold data were obtained for all stimuli used in the experiment. The background field upon which the stimuli were presented was such that the average exposure duration recognition threshold for hues would be no higher than 
for hue names, so that if expected shorter RTs to hue names were obtained the difference could not be attributed directly to recognition threshold differences.

In addition to investigating variations in mean RT as functions of stimulus type and number of potential alternatives, an attempt was made to determine effects of these variables on hypothetical component latencies underlying the RTs. One of several approaches aimed at identifying some of these component latencies has been to make inferences about them from observations of statistical distributions of total RTs (e.g., McGill, 1963). Recent studies reported by Hohle $(1965,1967)$ have established the tenability of a hypothesis that one of the component latencies may be represented by a random variable with an exponential distribution, and the sum of those remaining by a normally distributed random variable. The present experiment included a further investigation of these inferred components of RT, and an attempt was made to relate them to the independent variables.

\section{EXPERIMENT 1}

\section{Method}

Subjects. The Ss, paid volunteers, were six experimentally naive high school girls, 16 years of age.

Apparatus and stimulus materials. The stimuli were presented in a two-field tachistoscope, with the reaction stimuli presented on the primary field and the list of potential stimuli, viewed between trials, located on the secondary field. A trial began with a click signifying to $S$ that the reaction stimulus would appear in $750 \mathrm{msec}$. Following the $750 \mathrm{msec}$ foreperiod the primary field of the tachistoscope, containing the reaction stimulus, was switched on, and a chronoscope was started stimultaneously. The S's verbal response (naming the stimulus) terminated the stimulus and stopped the chronoscope by activating a voice key. A repeat cycle timer began a new trial every $5.2 \mathrm{sec}$ by simultaneously resetting the chronoscope and activating a timer that initiated the $750 \mathrm{msec}$ foreperiod.

The stimulus hues and hue names included pink, blue, white, green, yellow, and red. Both the hues (1/2 $\times 1 / 8$ in. rectangles, the size of a five-letter word in capitalized pica type) and corresponding hue names (black capitalized pica type) were presented on identical light gray backgrounds.

Design and procedure. Each $\mathrm{S}$ was presented two-, four-, and six-item lists in each of 10 consecutive sessions in which RT data were collected. Only hues or hue names were presented in a given session, with alternation over sessions. Within each session the two-, four-, and six-item lists were presented in separate blocks of trials with block order counterbalanced between Ss within sessions and within Ss over days. For the multiple stimulus lists the stimuli within a given block (list size) were presented in a quasi-random sequence, with each stimulus appearing 20 times. One of the six hues and correspond- ing hue names was randomly assigned to each $S$ and all sessions began with 20 warm-up trials on the appropriate one of these. Each list size for a given $S$ included the stimuli in the next smaller list. No two Ss were assigned the same set of stimuli in the one-, two-, or four-item lists, but the six stimulus list was the same for all six Ss. Since the different list sizes were all presented each session, the exact list of potential stimuli for a given block was kept in S's view between trials.

In Sessions 11 and 12, recognition threshold data were obtained by the method of constant stimuli. Each of the six hues and hue names was presented eight times at each of five exposure durations $(6.3,8.4$, $11.1,12.8$, and $15.5 \mathrm{msec}$ ) each session. Half the Ss began Session 11 with hues and half began with hue names. Order of stimulus presentation and exposure duration was randomly determined. In all 12 sessions a $30 \mathrm{sec}$ rest period followed each 40 trials.

Four measures were derived from each S's distributions of 20 RTs per stimulus per day at each level of list length: (1) mean RT; (2) parameter a, representing an estimated mean of an inferred exponentially distributed component latency; (3) parameter $b$, representing an estimated mean of an inferred normally distributed component; and (4) parameter c, representing an estimate of the standard deviation of the normal component (see Hohle, 1965, 1967). The parameters a, $b$, and $c$ were estimated by the method of moments as described by Hohle (1967). Wherever routine application of this method resulted in a negative estimate for a parameter, this value was arbitrarily set equal to zero. Data obtained for the two stimuli common to the two-, four-, and six-item lists for each S were analyzed. The stimulus that occurred in the one-stimulus condition as well as in the multiple-stimulus lists for a given $\mathrm{S}$ was designated the familiar stimulus.

A four factor analysis of variance was repeated with mean RT and each of the parameters, $a, b$, and $c$ as dependent measures. For each of these measures, the four treatments, all of which were within-Ss treatments, were Days, List length, Familiar vs Novel, and Hues vs Hue Names. A 5\% significance level was adopted to assess statistical significance of all effects examined.

\section{Results}

Mean $R T$. There was a significant decrease in mean RT across days, $(F=3.78$, df $=4 / 20, p<.05)$, and a significant list length by hues vs hue names interaction $(F=56.94, d f=2 / 10, p<.05$; see Figs. $1 i$ and $2 i)$. Supplementary analyses of variance indicated significant list length effects for both hues $(F=97.52$, $\mathrm{df}=$ $2 / 10, p<.05)$ and hue names $(F=8.81, d f=2 / 10, p<.05)$. Further supplementary analyses of variance of hues vs hue names at each level of list length showed that they differed significantly at list length four $(F=10.45$, $\mathrm{df}=1 / 5, \mathrm{p}<.05)$ and $\operatorname{six}(\mathrm{F}=43.13, \mathrm{df}=1 / 5, \mathrm{p}<.05)$ but not at list length two.

The list length by familiar vs novel interaction was 

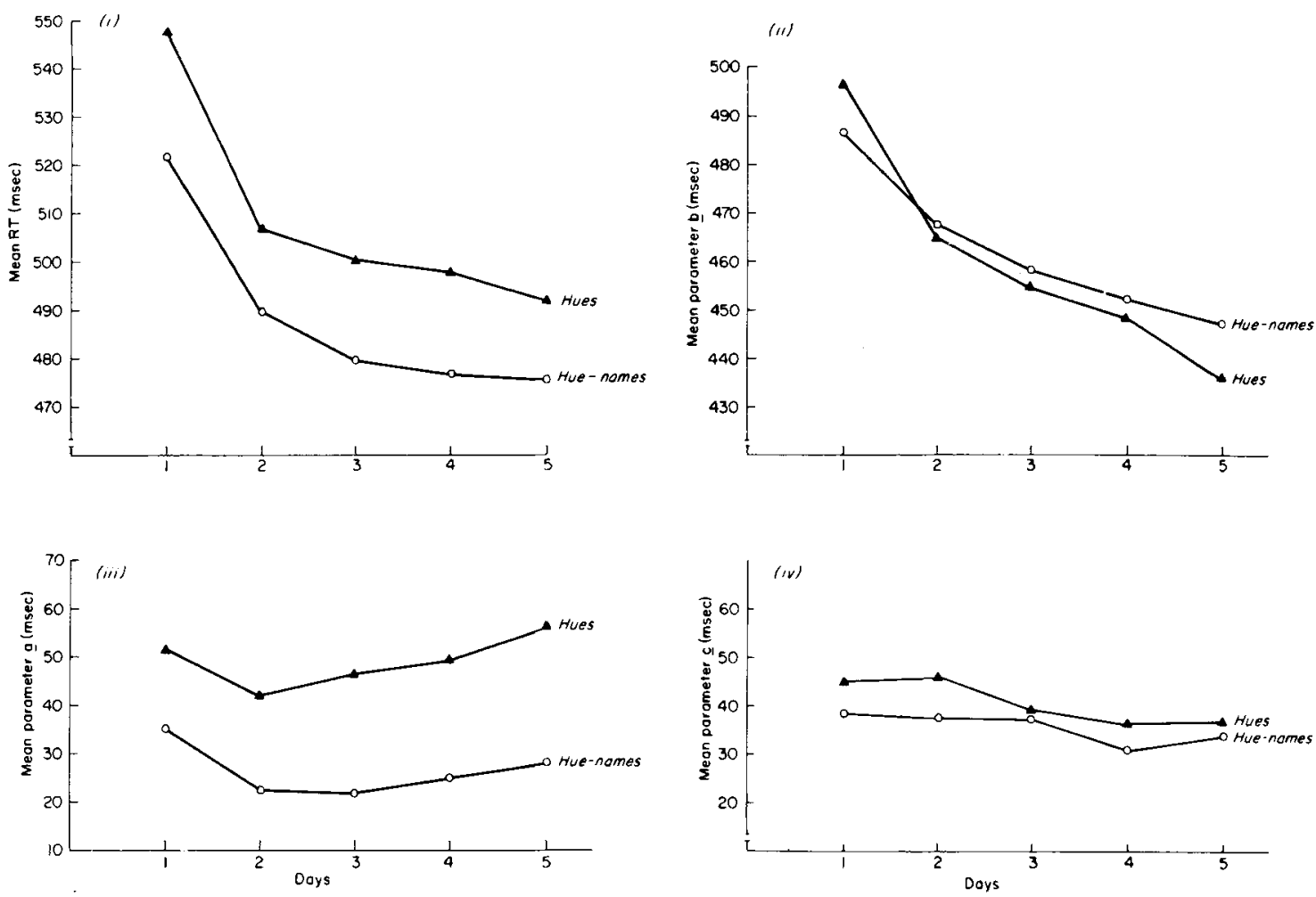

Fig. 1. Mean choice RT and inferred components of RT as functions of days of practice with hues and hue names as stimuli.

also significant $(F=5.54, d f=2 / 10, p<.05)$, but, as will be indicated below, the comparable interaction with forms and form names as stimuli was not significant.

Parameter a. There was a slight but significant overall increase in parameter a with increasing list length $(F=8.59, \mathrm{df}=2 / 10, p<.05)$, and the mean value of this parameter was greater for hues than for hue names $(F=23.67, \mathrm{df}=1 / 5, \mathrm{p}<.05$; see Fig. 2iii). The familiar vs novel by hues vs hue names interaction was also significant $(F=8.11, \mathrm{df}=1 / 5, \mathrm{p}<.05)$, but, as was true for mean $\mathrm{RT}$, a corresponding interaction was not found when the stimuli were forms vs form names (see below). Mean values of a did not vary significantly as a function of days of practice $(F=2.04$, $\mathrm{df}=4 / 20, \mathrm{p}>.05)$.

Parameter $b$. In contrast to parameter $a$, the mean value of $b$ decreased significantly over days of practice $(F=4.34, d f=4 / 20, p<.05$; see Fig. 1ii). There was also a marked interaction (Fig. 2ii) of list length by hues vs hue names $(F=15.59, d f=2 / 10, p<.05)$. Parameter $b$ was lower for hues at list length two, but this relationship was reversed at the longer list lengths. Follow-up analyses of variance indicated that $b$ increased with list length when hues were stimuli $(F=35.25, \mathrm{df}=2 / 10, \mathrm{p}<.05)$, but not when hue names were stimuli $(F=3.61, \mathrm{df}=2 / 10, p>.05)$.

Parameter $c$. A significant list length by hues vs hue names interaction was obtained $(F=10.12, d f=2 / 10$, $\mathrm{p}<.05$ ) for this parameter (see Fig. 2iv). Follow-up analyses of variance indicated the slight decrease in c with list length was significant when hue names were stimuli $(F=7.27, d f=2 / 10, p<.05)$, but there was no change when hues were stimuli $(F=2.04, d f=2 / 10$, p> .05). This parameter was significantly smaller for hue names than hues only. at list length four ( $F=9.41$, $\mathrm{df}=1 / 5, p<.05)$. The mean values of $c$, shown in Fig. 1iv, did not vary over days of practice.

Exposure duration threshold data. Tabulation of correct and incorrect recognition responses for hues and hue names indicated that all six ss made more errors when the stimuli were hue names than when they were hues, and five out of six Pearson chi square statistics computed from individual Ss' data were significant at the .05 level. It was concluded, therefore, that the mean exposure duration recognition thresholds were higher for hue names than for hues.

\section{EXPERIMENT 2}

In order to investigate the generality of the object vs object name results of Experiment 1, a second experiment using forms vs form names as stimuli was carried out. Fraisse (1960) reported a study in which verbal identification RTs to four geometrical figures and the corresponding figure names were obtained from five Ss. Median time for naming the words was significantly shorter than time for naming the figures.

\section{Method}

Subjects. The Ss, paid volunteers, were six 12- 


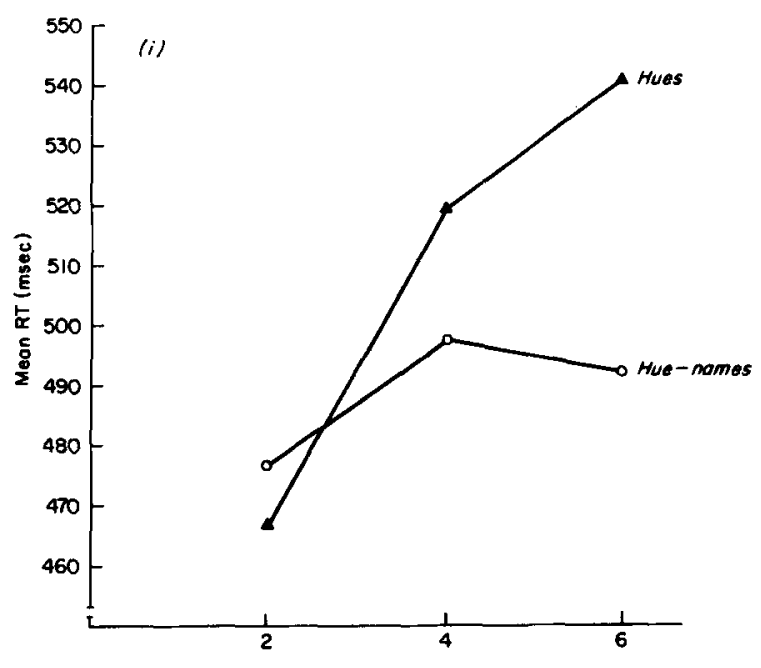

(ii)
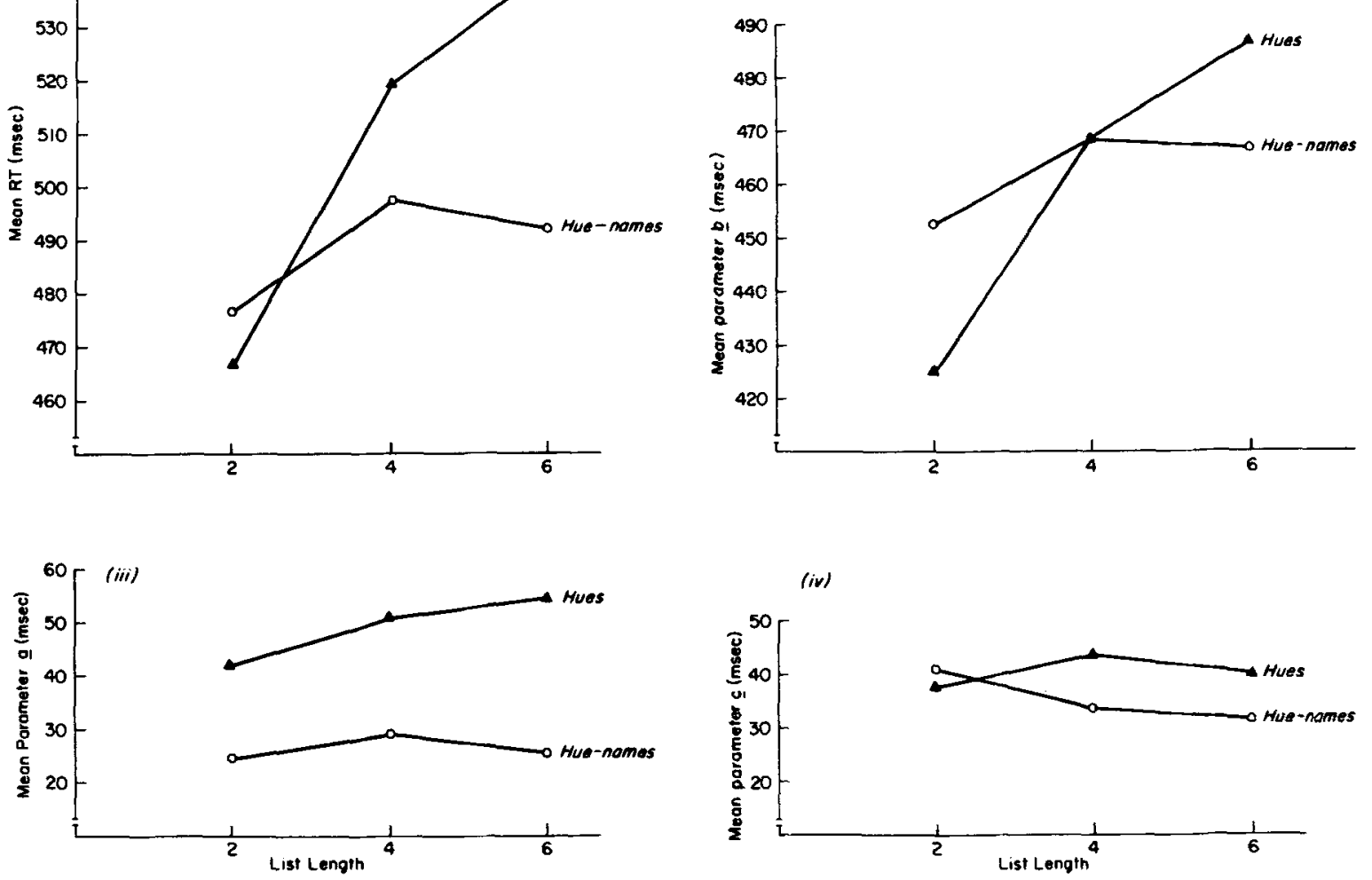

Fig. 2. Mean choice RT and inferred components of RT as functions of list length (size of the stimulus set) with hues and hue names as stimuli.

year-old schoolgirls. Five of them had participated in a choice RT study in which numerals were stimuli about six months earlier.

Stimuli. The form stimuli were cut out of solid black construction paper and pasted on white background fields. The forms and areas were as follows: square, $098 \mathrm{sq}$. in.; rectangle, .094 sq. in.; circle, .093 sq. in.; ellipse, .098 sq. in.; triangle, .095 sq. in.; and diamond, $.102 \mathrm{sq}$, in. The corresponding form names were printed in black capitalized pica type on fields identical to those on which the forms were presented.

Apparatus, design, and procedure. Except for those differences in Ss and stimuli noted above, this experiment was identical in every respect to Experiment 1.

Four measures (i.e., mean RT, and parameters a, $b$, and c) were again derived from each S's distributions of 20 RTs per stimulus per day at each level of list length. Analysis of variance, performed on each dependent measure, included four within-Ss treatments: Days, List length, Familiar v8 Novel, and Forms vs Form Names.

\section{Results}

Mean $R T$. There was a significant forms $\mathbf{v s}$ form names by list length interaction $(F=14.12$, $d f=2 / 10$, $p<.05$; see Fig. $3 i$ ). A three factor analysis of variance of forms vs form names at each level of list length showed; consistent with Experiment 1, that RTs were significantly smaller for form names than forms at list lengths four $(F=129.76, d f=1 / 5, p<.05)$ and $\operatorname{six}$ $(F=84.85, \mathrm{df}=1 / 5, \mathrm{p}<.05)$, but not at list length two. Further supplementary analyses of variance indicated, also consistent with Experiment 1, significant list length effects for both forms $(F=13.22$, $d f=2 / 10$, $p<.05)$ and form names $(F=28.39, d f=2 / 10, p<.05)$. In contrast to Experiment 1, however, neither the main effect of days nor the familiar vs novel by list length interaction was significant.

Parameter $a$. The mean value of this parameter was greater for forms than form names $(F=40.48, \mathrm{df}=1 / 5$, $\mathrm{p}<.05$ ) as can be seen graphically in Fig. 3ili). This result was consistent with the hues vs hue names difference obtained in Experiment 1. Contrary to results of Experiment 1, however, neither the familiar vs novel by forms vs form names interaction nor the main effect of list length was significant in this study.

Parameter $b$. Consistent with Experiment 1, the forms vs form names by list length interaction was significant $(\mathrm{F}=19.16, \mathrm{df}=2 / 10, \mathrm{p}<.05)$. These means are plotted in Fig. 3ii. Follow-up analyses of variance indicated that $b$ increased with list length for both forms ( $F=$ 29.29, $\mathrm{df}=2 / 10, \mathrm{p}<.05)$ and form names $(\mathrm{F}=\mathbf{2 3 . 5 5}$, 

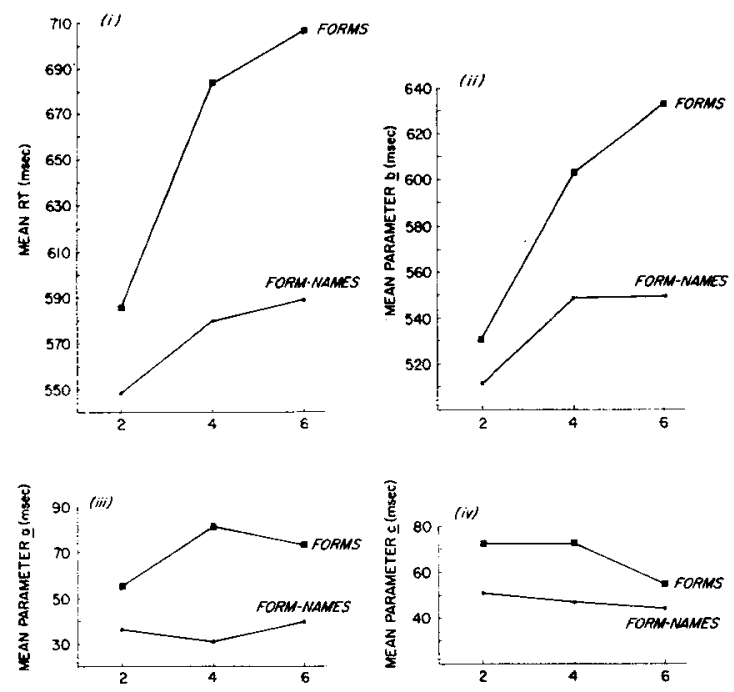

Fig. 3. Mean choice RT and inferred components of RT as functions of list length (size of stimulus set) with forms and form names as stimuli.

$\mathrm{df}=2 / 10, \mathrm{p}<.05$; $\mathrm{cf}$. Experiment 1, where the list length effect for hue names was not significant).

Parameter $c$. The mean value of this parameter was smaller for form names than for forms ( $F=28.91$, $\mathrm{df}=1 / 5, \mathrm{p}<.05)$, and it decreased significantly with increases in list length $(F=8.00, d f=2 / 10, p<.05)$. Mean values of $c$ are plotted in Fig. 3iv.

\section{DISCUSSION}

This section will be concerned primarily with object vs object name differences that were consistent in the two studies described above. The slight differences in results between the two experiments may have resulted from any one or all of the following: age differences of Ss (16- vs 12-year-olds), stimulus differences (hues and hue names vs forms and form names), or sampling error.

\section{Mean RT}

The significant effect of days in Experiment 1, indicating decrease in mean RT across sessions, was in accord with earlier findings of Brown (1915) and Lund (1927). The Ss in Experiment 2 had previously participated in a similar study, and this may have been enough experience to eliminate practice effects.

The overall greater mean RTs for objects than for object names cannot be accounted for in terms of recognition thresholds in either study. In Experiment 1 the duration thresholds for recognition of hues were significantly lower than for hue names, as described above, and in Experiment 2 the forms used as stimuli could be identified without error at exposure durations well below those at which errors were made in identifying the form names.

The list length by stimulus type interactions obtained in both stidues (see Figs. $2 i$ and 3i) indicated that at list lengths four and six the RTs for names were smaller

than for objects-which is consistent with earlier findings (e.g., Brown, 1915; Schiller, 1966)-but no differences were obtained at list length two (the obtained means were actually reversed in Experiment 1 ). These interactions apparently would not be predicted by analyses offered by earlier investigators (who were directly concerned only with hues vs hue names) as follows: (a) the "distinct physiological processes" hypothesis (Brown, 1915) does not appear to suggest that differences between the physiological processes for the two types of stimuli should increase with increases in list length; (b) the "differential amounts of practice" hypothesis (Lund, 1927) suggests that differential frequency of verbalization is the factor accounting for the difference between the two functions, but the amount of differential practice for a given object vs the corresponding object name would be the same regardless of the length of list in which the stimuli appeared; (c) the "common plus special factors" hypothesis offered by Ligon (1932) suggests that the special factor for a given object is larger by some finite amount than the special factor for the corresponding object name and does not suggest that the difference between the two special factors should change with number of potential alternatives; and (d) the "coding" hypothesis (Schiller, 1966) suggests that words provide a more efficient medium for coding than objects due to redundancy of letters and parts of words, and does not appear to predict differential coding efficiency with changes in list length. A fifth interpretation, the "response competition" hypothesis (Stroop, 1935, 1938), suggests more nonverbal responses are associated with a given object than with the corresponding object name, and the nonverbal responses interfere with the response of naming the object. This hypothesis could predict the interactions reported here if it were assumed that adding an object to the stimulus ensemble added more competing responses than adding the corresponding object name.

Although any of the hypotheses cited above would predict the results obtained in this study at either list length four or six, none of them, with the possible exception of the response competition interpretation, appear to predict the objects vs object names interactions. Another possible means of accounting for the results of the present studies is that there may have been greater interstimulus interference due to stimulus generalization among the objects than among the object names: increasing the number of hues or forms in the stimulus set would then be expected to lead to a greater increase in mean RT than a corresponding increase in the number of hue names or form names. That mean RTs were faster for hues than hue names in the twoitem list may have been due to the lower recognition thresholds for hues (cf. results of Kaswan \& Young, 1965), whereas this effect was overridden by greater interstimulus interference among hues with the longer list lengths. 


\section{Paramefer a}

The finding that parameter a was considerably smaller for hue names than hues (Experiment 1) and form names than forms (Experiment 2) may indicate that this parameter is a measure of an exponentially distributed "response choice latency" as proposed by Christie and Luce (1956). It might be assumed that one response habit, that of naming, has been associated with a given object name while a variety of responses, both verbal and nonverbal have been associated with a given object. This view has been discussed by Morin, Konick, Troxell, and McPherson (1965). In making a decision to respond it might then be necessary for $S$ to scan a greater number of response associates when objects were stimuli than when object names were stimuli. One problem with this view is that it appears to suggest a practice effect or a days by objects vs object names interaction, since the required response would be expected to become more dominant with practice. But the Ss spent only about $35 \mathrm{~min}$ per day in the experimental setting, and between-sessions practice might have eliminated expected response dominance: the Ss were probably responding in a variety of ways to the objects and object names outside the experimental setting.

The finding of a slight but significant increase in parameter a with increase in list length in Experiment 1 was not replicated in Experiment 2, nor was it obtained in a related study reported elsewhere (Gholson \& Hohle, in press).

\section{Parameter b}

Since the sum of parameters $a$ and $b$ is equal to mean $\mathrm{RT}$, the finding in Experiment 1 that $\mathrm{b}$ decreased with practice while a did not indicates that the practice effect found for mean RT is completely accounted for by effects of practice on the inferred normally distributed component of RT.

The list length by stimulus type interactions obtained in both studies paralleled similar interactions obtained for results of mean RT analyses. Thus these interaction effects on mean RT can be attributed more specifically to effects on parameter $b$, that is, to effects on an inferred normally distributed component of RT.

\section{Parameter c}

Results for this parameter were not completely consistent but generally appear to indicate $\mathrm{c}$ is smaller for object names than objects and decreases slightly in its mean value with increases in listlength. Speculation concerning these findings appears premature and will have to await further understanding of the component processes of $\mathrm{RT}$.

\section{References}

Brown, W. Practice in associating color-names with colors Psychol. Rev., 1915, 22, 45-55

Christie, R., \& Luce, R. Decision structure and time relations in simple choice behavior. Bull. math. Bioph., 1956, 18, 89-112.

Comalli, P. E., Jr., Wapner, S., \& Werner, M. Interference effects on Stroop color word test in childhood, adulthood, and aging. J. Genet. Psychol., 1962, 100, 47-53.

Fraisse, P. Recognition time measured by verbal reaction to figures and words. Percept. mot. Skills, 1960, 11, 204.

Gholson, B., \& Hohle, R. H. Choice reaction times to hues printed in conflicting hue names and nonsense words. J. exp. Psychol., 1968, (in press).

Hohle, R. H. Inferred components of reaction times as functions of foreperiod duration. J. exp. Psychol., 1965, 69, 382-386.

Hohle, R. H. Component process latencies in reaction times of children and adults. In L. P. Lipsitt \& C. C. Spiker (Eds.), Advances in child development and behavior. Vol. III. New York: Academic Press, 1967.

Kaswan, J., \& Young, S. Effect of stimulus variables on choice reaction times and thresholds. J. exp. Psychol., 1965, 69, 511514.

Ligon, E, M. A genetic study of color naming and word naming. Amer. J. Psychol., 1932, 44, 103-122.

Lund, F. H. The role of practice in speed of association. J. exp Psychol., 1927, 10, 424-433.

McGill, W. J. Stochastic latency mechanisms. In R. D. Luce, R. R. Rush, \& E. Galanter (Eds.), Handbook of mathematical psychology. New York: Wiley, 1963.

Morin, R. E., Konick, N., Troxell, N., \& McPherson, S. Information and reaction time for "naming" responses. J. exp. Psychol., $1965,70,309-314$

Schiller, P. Developmental study of color-word interference. $J$. exp. Psychol, 1966, 72, 105-108.

Stroop, J. R. Studies of interference in serial verbal reactions. $J$ exp. Psychol, 1935, 18, 643-662.

Stroop, J. R. Factors affecting speed in serial verbal reactions. Psychol. Monogr., 1938, 50, 38-48.

\section{Notes}

1. Part of this paper is based on a thesis submitted by the first author in partial fulfillment of the requirements for the M.A. degree at the University of Iowa.

2. Data analysis reported in this paper were carried out using the facilities of the University of Iowa Computer Center. Funds for this purpose were provided by a grant from the Graduate College of the University of Iowa, and by United States Public Health Research Grant HD 0261-01.

(Accepted for publication November $5,1967$. 